\title{
The Effects of Hepatitis C Treatment Eligibility Criteria on All-cause Mortality Among People With Human Immunodeficiency Virus
}

\author{
Alexander Breskin, ${ }^{1}$ Daniel Westreich, ${ }^{1}$ Christopher B. Hurt, ${ }^{2}$ Stephen R. Cole, ${ }^{1}$ Michael G. Hudgens, ${ }^{3}$ Eric C. Seaberg, ${ }^{4}$ Chloe L. Thio, ${ }^{5}$ Phyllis C. Tien, ${ }^{6}$ and \\ Adaora A. Adimora ${ }^{1,2}$ \\ ${ }^{1}$ Department of Epidemiology, ${ }^{2}$ Institute for Global Health and Infectious Diseases, and ${ }^{3}$ Department of Biostatistics, University of North Carolina at Chapel Hill; Departments of ${ }^{4}$ Epidemiology, and \\ ${ }^{5}$ Medicine, Johns Hopkins University, Baltimore, Maryland; and ${ }^{6}$ Department of Medicine, University of California, San Francisco
}

Background. The cost of direct-acting antivirals (DAAs) for hepatitis C virus (HCV) prompted many payers to restrict treatment to patients who met non-evidence-based criteria. These restrictions have implications for survival of people with HCV, especially for people with human immunodeficiency virus (HIV)/HCV coinfection who are at high risk for liver disease progression. The goal of this work was to estimate the effects of DAA access policies on 10-year all-cause mortality among people with HIV.

Methods. The study population included 3056 adults with HIV in the Women's Interagency HIV Study and Multicenter AIDS Cohort Study from 1 October 1994 through 30 September 2015. We used the parametric g-formula to estimate 10-year all-cause mortality under DAA access policies that included treating (i) all people with HCV; (ii) only people with suppressed HIV; (iii) only people with severe fibrosis; and (iv) only people with HIV suppression and severe fibrosis.

Results. The 10-year risk difference of treating all coinfected persons with DAAs compared with no treatment was $-3.7 \%$ (95\% confidence interval [CI], $-9.1 \%$ to $.6 \%)$. Treating only those with suppressed HIV and severe fibrosis yielded a risk difference of $-1.1 \%$ (95\% CI, $-2.8 \%$ to $.6 \%$ ), with $51 \%$ (95\% CI, 38\%-59\%) of coinfected persons receiving DAAs. Treating a random selection of $51 \%$ of coinfected persons at baseline decreased the risk by $1.9 \%$ ( $95 \% \mathrm{CI},-4.7 \%$ to .3\%).

Conclusions. Restrictive DAA access policies may decrease survival compared to treating similar proportions of people with $\mathrm{HIV} / \mathrm{HCV}$ coinfection with DAAs at random. These findings suggest that lives could be saved by thoughtfully revising access policies.

Keywords. hepatitis $\mathrm{C}$ virus; human immunodeficiency virus; antiretroviral therapy; direct-acting antivirals; population intervention effects.

Given the many comorbidities associated with human immunodeficiency virus (HIV), understanding the impact of interventions for these conditions is essential for allocating patient, clinician, and public resources. Liver-related complications are a major cause of death among people with HIV (PWH), largely due to hepatitis $\mathrm{C}$ virus (HCV) coinfection $[1,2]$. Fortunately, the advent of direct-acting antiviral (DAA) treatments has dramatically changed the outlook for people with HIV/HCV coinfection; sustained HCV virologic response (SVR) is achievable in $>90 \%$ of $\mathrm{PWH}$ [3].

To limit expenditures, payers have restricted DAA treatment to those who meet certain criteria. As of October 2018, 15 Medicaid programs restricted DAA treatment based on liver fibrosis, with 7 limiting access to those beneficiaries at or nearing

Received 15 August 2018; editorial decision 27 December 2018; accepted 4 January 2019; published online January 7, 2019.

Correspondence: A. Breskin, Department of Epidemiology, University of North Carolina at Chapel Hill, CB 7435 McGavran-Greenberg Hall, Chapel Hill, NC 27599 (abreskin@unc.edu).

Clinical Infectious Diseases ${ }^{\circledR} \quad$ 2019;69(9):1613-20

(C) The Author(s) 2019. Published by Oxford University Press for the Infectious Diseases Society of America. All rights reserved. For permissions, e-mail: journals.permissions@oup.com. DOI: $10.1093 /$ cid/ciz008 cirrhosis [4]. Achievement of HIV benchmarks for persons with HIV/HCV coinfection, such as suppressing HIV viremia, has been used as a restriction for DAA treatment in 5 states plus Washington, District of Columbia [5]. These restrictions persist and treatment denials remain common [6] despite guidelines recommending DAA treatment for nearly all patients with HCV [7]. Because these policies are evolving [8], evidence is needed to properly evaluate how such restrictions impact mortality-especially for people with $\mathrm{HIV} / \mathrm{HCV}$ coinfection who are at elevated risk for liver disease progression [9].

Our goal was to evaluate the impact of restrictions for DAA use on 10-year, all-cause mortality among PWH at 2 levels. First, we estimated the effects of various DAA treatment policies on mortality among HIV/HCV-coinfected persons to determine how much mortality could be averted by expanding access to DAAs. Second, we estimated the population-level impact of these criteria on mortality among the entire population of PWH. These estimates consider the prevalence of $\mathrm{HCV}$ among $\mathrm{PWH}$, and thus facilitate comparisons of HCV treatment interventions with interventions for other comorbidities. Together, these results provide an important perspective 
that may be used by policymakers when setting future HCV treatment criteria for PWH.

\section{METHODS}

\section{Study Sample}

Our data came from ongoing, US-based HIV cohorts of men who have sex with men (Multicenter AIDS Cohort Study [MACS]) [10] and women (Women's Interagency HIV Study [WIHS]) [11, 12]. MACS began enrollment in 1984, with additional recruitment in 1987, 2001, and 2010 in 4 cities. WIHS opened in 1994 in 6 cities and added additional participants in 2001, 2011, and 2013; it now represents 10 urban and suburban locations. In both cohorts, participants attend semiannual visits for laboratory testing, clinical examinations, and interviews. Information collected at these visits includes medication use, sociodemographic and behavioral characteristics, CD4 cell count and HIV RNA, and HCV antibody and RNA. Additionally, scores assessing liver fibrosis (Fibrosis-4 Index for Liver Fibrosis [FIB-4] [13] and aspartate aminotransferaseto-platelet ratio index [APRI] [14]) are calculated for all participants. The WIHS and MACS were approved by the institutional review boards of participating institutions, and all participants provided informed consent.

Eligibility for this analysis required documentation of HIV infection at cohort entry or HIV seroconversion during follow-up (with inclusion beginning at the visit corresponding to HIV diagnosis). Only visits after the start of WIHS recruitment (1 October 1994) were included. All participants were required to be antiretroviral therapy (ART) naive (defined below) and free of an AIDS diagnosis at their first eligible visit. Follow-up lasted until the first of: loss to follow-up (the date of a participant's second consecutive missed study visit), death, 10 years after the first eligible visit, or 30 September 2015.

\section{Definitions}

The presence of antibody $(\mathrm{Ab})$ against $\mathrm{HCV}$ was tested at baseline in both studies by enzyme immunoassay. Reactive specimens underwent HCV RNA testing by nucleic acid amplification; those with detectable HCV RNA were considered to have active, chronic HCV infection. Participants who lacked $\mathrm{HCV}$ RNA tests but had reactive $\mathrm{HCV}$ Ab were considered to have missing HCV data.

The definition of ART was guided by the November 2014 US Department of Health and Human Services guidelines [15]. Any 3-drug regimen including at least 1 protease inhibitor, entry inhibitor, integrase inhibitor, or nonnucleoside reverse transcriptase inhibitor was considered ART. Once a participant initiated ART, they were assumed to remain on it for the remainder of follow-up. ART initiated after 1 October 2001 (the first visit following the approval of tenofovir, a component of many current ART regimens [16]) was considered "modern ART."
Date and cause of death were identified either directly through periodic searches of the National Death Index or through copies of death certificates obtained by study investigators.

\section{Confounders}

Confounders for the ART-on-mortality and HCV-onmortality relationships were chosen using a causal diagram [17] (Supplementary Figure 1). Baseline confounders were age, sex, race/ethnicity, CD4 count, HIV RNA, injection drug use (IDU), heavy alcohol use (defined as $>7$ drinks per week for women and >14 drinks per week for men [18]), smoking, obesity, and liver fibrosis. Time-varying confounders for the ARTon-mortality relationship included CD4 count, HIV RNA, IDU, heavy alcohol use, smoking, obesity, and liver fibrosis. Fibrosis was categorized into 3 levels: FIB- $4 \geq 3.25$ or APRI $\geq 1$ was classified as severe fibrosis/cirrhosis, whereas FIB-4 $<1.45$ and APRI $<0.7$ (together) was classified as no significant fibrosis. Other combinations were considered moderate fibrosis. The APRI cutoffs were guided by a meta-analysis [14] that suggested these perform better than commonly used cutoffs of 2 and 0.5 , respectively. Details on variable measurement and operationalization are shown in the Supplementary Appendix.

\section{Statistical Analysis}

We compared the risk of 10 -year, all-cause mortality among $\mathrm{HIV} / \mathrm{HCV}$-coinfected persons and their risk under DAA access policies including treating $(i)$ all $\mathrm{HIV} / \mathrm{HCV}$-coinfected persons; (ii) HIV/HCV-coinfected persons who meet certain preconditions (HIV suppression, severe fibrosis or cirrhosis, or both); and (iii) treating the same proportion of HIV/HCV-coinfected persons as in (ii) chosen randomly without clinical restrictions. Additionally, we estimated the population intervention analogues of the above effects that compare mortality among all PWH to mortality had HIV/HCV-coinfected persons been treated according to each of the criteria [19-21]. Each effect was estimated under a hypothetical intervention to have all participants initiate modern ART at baseline, including those who entered the study prior to 2001. Each effect is defined in detail in the Supplementary Appendix.

Because insufficient person-time has accrued in the DAA era to estimate long-term effects, the above effects cannot be directly estimated with existing data. Instead, we used data collected prior to the DAA era along with strong assumptions to estimate the effects in this study. Specifically, we assumed that an HCV-seronegative person has the same risk of mortality as a person with HCV after successful treatment of their HCV infection, provided they both have similar degrees of liver fibrosis and are of similar age, race and ethnicity, sex, smoking status, etc. We conducted a sensitivity analysis restricted to those negative for hepatitis B virus (HBV) surface antigen at baseline, as $\mathrm{HBV}$ is known to be associated with liver fibrosis and mortality, and thus may lead to a violation of this assumption. 
We estimated the mortality risk under each policy using the parametric g-computation algorithm (referred to as g-computation) [22]. The g-computation is an extension of direct standardization that accommodates time-varying confounders and dynamic treatment regimens [23]. Our implementation involves conducting multiple microsimulations within a single, enlarged cohort, with each microsimulation corresponding to a different treatment policy $[24,25]$. The enlarged cohort consisted of 50000 individuals randomly sampled with replacement from the eligible MACS/WIHS participants. We used pooled regressions to model mortality and the time-varying confounders in the MACS/WIHS. Using these models, we simulated the time-varying confounder histories and survival curves that would occur in the enlarged cohort under each DAA policy, and we compared 10-year mortality for each policy. We assumed that DAAs had an average effectiveness of $96 \%$ [26]. Confidence limits were estimated using the nonparametric bootstrap percentile method with 1000 replicates [27]. Further details of g-computation are shown in the Supplementary Appendix.

We used multiple imputation to handle missing baseline data with a multivariate normal imputation model [28]. The proportion missing for each variable ranged from $0 \%$ to $30 \%$ (for fibrosis, specifically). Missing time-varying covariates were carried forward from the most recent measurement. The results remained similar when linear interpolation was used for missing time-varying covariates. We incorporated multiple imputation into the bootstrap with the "Boot MI" algorithm [29] with 20 imputed datasets per replicate. All statistical analyses were conducted with SAS version 9.4 software (SAS Institute, Cary, North Carolina).

\section{RESULTS}

\section{Study Sample}

A total of $3056 \mathrm{PWH}$ were included in the study (1777 women, 1279 men). The prevalence of HCV was 18\% (27\% among women; $7 \%$ among men). Follow-up lasted a median of 7.5 years (interquartile range, 2-10 years). Those with HIV/HCV coinfection were generally older; were more likely to be female; had lower CD4 counts; were more likely to use injection drugs, drink heavily, or smoke; and had worse fibrosis at baseline (Table 1). ART was prescribed to $63 \%$ of participants, $32 \%$ of whom initiated a modern ART regimen. The observed and modeled distributions of key variables were similar (Supplementary Table 1 and Supplementary Figure 2).

\section{Estimated Effects of DAA Access Criteria}

The risk difference (RD) comparing 10-year all-cause mortality risk among coinfected persons who were not treated for $\mathrm{HCV}$ to what would be expected if all were treated with DAAs was $-3.7 \%$ (95\% confidence interval [CI], $-9.1 \%$ to .6\%; Table 2). This corresponds to a 10 -year number needed to treat (NNT) of
27, meaning coinfected individuals would need to receive DAA treatment to prevent 1 death over 10 years.

If only those coinfected persons with undetectable HIV RNA received DAAs, mortality risk would be reduced by $-1.9 \%$ (95\% $\mathrm{CI},-5.2 \%$ to $.5 \%$ ), for a 10 -year NNT of 52 (meaning that this policy must be applied to 52 coinfected persons to prevent 1 death over 10 years [30]). Under this criterion, 90\% (95\% CI, $84 \%-93 \%$ ) of coinfected persons would be treated with DAAs. The effect of treating the same proportion of coinfected persons at baseline without regard for HIV viral suppression (that is, the effect of randomly choosing $90 \%$ of coinfected persons at baseline to receive treatment) was an expected mortality risk reduction of $-3.3 \%$ ( $95 \% \mathrm{CI},-8.1 \%$ to $.5 \%)$, for a 10 -year NNT of 30 (Figure 1 and Table 2).

Only providing DAAs to coinfected persons with severe fibrosis or cirrhosis would be expected to reduce the risk of mortality by $-1.6 \%$ ( $95 \% \mathrm{CI},-4.7 \%$ to $.8 \%)$, and $60 \%(95 \%$ CI, $45 \%-70 \%$ ) of coinfected persons would receive treatment. The corresponding 10 -year NNT is 62 . If the same proportion of coinfected persons were randomly selected for treatment at baseline, mortality risk would be reduced by $-2.2 \%$ (95\% CI, $-5.6 \%$ to .3\%), for a 10 -year NNT of 45 (Figure 1 and Table 2).

Finally, by only treating coinfected persons with severe fibrosis or cirrhosis who have undetectable HIV RNA, the risk of mortality would be expected to decline by $-1.1 \%$ (95\% CI, $-2.8 \%$ to $.6 \%$ ), and $51 \%$ (95\% CI, $38 \%-59 \%$ ) of coinfected persons would be treated. This translated into a 10 -year NNT of 94 . By treating the same proportion of coinfected persons at baseline without these restrictions, mortality would be expected to decline by $-1.9 \%$ ( $95 \% \mathrm{CI},-4.7 \%$ to $.3 \%$; Figure 1 and Table 2), and the NNT is 53. The timing of DAA treatments under each set of treatment criteria is presented in Supplementary Figure 3. Though the magnitude of the effects estimated after excluding those with $\mathrm{HBV}$ at baseline are somewhat larger than in the main analysis, the overall pattern of the results remains the same (Supplementary Table 2). The amount of person-time on treatment for each policy is displayed in Supplementary Table 3.

\section{Estimated Population Intervention Effects of DAA Access Criteria}

The $\mathrm{RD}$ comparing the 10-year all-cause mortality risk in the entire population of $\mathrm{PWH}$ to the risk in the entire population that would be expected if all coinfected persons received DAAs was $-0.7 \%$ ( $95 \% \mathrm{CI},-1.8 \%$ to $.1 \%$; Table 3 ).

If only coinfected persons with undetectable HIV RNA received DAAs, the mortality risk among all $\mathrm{PWH}$ would be expected to be reduced by $-0.4 \%$ ( $95 \% \mathrm{CI},-1.0 \%$ to $.1 \%)$. In this scenario, $90 \%$ (the same proportion reported in the previous section) of coinfected persons received DAAs. The effect of treating the same proportion of coinfected persons randomly at baseline was an expected $-0.6 \%$ (95\% CI, $-1.6 \%$ to .1\%) decrease in mortality among all PWH (Table 3$)$. 


\begin{tabular}{|c|c|c|c|c|c|c|c|c|}
\hline \multirow{4}{*}{$\frac{\text { Characteristic }}{\text { Age, y, median (IQR) }}$} & \multicolumn{4}{|c|}{ Women's Interagency HIV Study } & \multicolumn{4}{|c|}{ Multicenter AIDS Cohort Study } \\
\hline & \multicolumn{2}{|c|}{$\mathrm{HCV}^{+}$} & \multicolumn{2}{|c|}{$\mathrm{HCV}^{-}$} & \multicolumn{2}{|c|}{$\mathrm{HCV}^{+}$} & \multicolumn{2}{|c|}{$\mathrm{HCV}^{-}$} \\
\hline & \multicolumn{2}{|c|}{$(N=460)$} & \multicolumn{2}{|c|}{$(N=1231)$} & \multicolumn{2}{|c|}{$(N=83)$} & \multicolumn{2}{|c|}{$(N=1180)$} \\
\hline & 40 & $(35-44)$ & 34 & $(29-40)$ & 44 & $(40-50)$ & 40 & (34-45) \\
\hline \multicolumn{9}{|l|}{ Race } \\
\hline White (non-Hispanic) & 74 & $(16.1)$ & 178 & (14.5) & 35 & $(42.2)$ & 802 & (68.0) \\
\hline African American & 281 & $(61.1)$ & 710 & $(57.7)$ & 40 & $(48.2)$ & 247 & (20.9) \\
\hline Hispanic & 99 & (21.5) & 293 & (23.8) & 8 & (9.6) & 116 & (9.8) \\
\hline Other & 6 & $(1.3)$ & 49 & $(4.0)$ & 0 & (0.0) & 15 & (1.3) \\
\hline Missing & 0 & & 1 & & 0 & & 0 & $\ldots$ \\
\hline CD4 count, cells/ $/ \mathrm{L}$, median (IQR) & 373 & $(213-575)$ & 410 & (262-609) & 405 & $(250-652)$ & 433 & $(280-605)$ \\
\hline Missing & 13 & $\ldots$ & 36 & $\ldots$ & 3 & $\ldots$ & 103 & $\ldots$ \\
\hline Detectable HIV RNA ${ }^{a}$ & 428 & (95.3) & 1137 & (94.3) & 59 & $(96.7)$ & 692 & (96.2) \\
\hline Missing & 11 & $\ldots$ & 25 & $\ldots$ & 22 & $\ldots$ & 461 & $\ldots$ \\
\hline \multicolumn{9}{|l|}{ IDU } \\
\hline Never & 61 & (13.3) & 1096 & $(89.1)$ & 41 & (52.6) & 1060 & (91.9) \\
\hline Former & 293 & (63.7) & 106 & (8.6) & 7 & $(9.0)$ & 56 & (4.9) \\
\hline Current & 106 & $(23.0)$ & 28 & (2.3) & 30 & (38.5) & 38 & (3.3) \\
\hline Missing & 0 & $\ldots$ & 1 & $\ldots$ & 5 & $\ldots$ & 26 & $\ldots$ \\
\hline Heavy alcohol use ${ }^{b}$ & 93 & (20.8) & 143 & (11.9) & 9 & $(11.7)$ & 109 & (9.5) \\
\hline Missing & 12 & $\ldots$ & 28 & $\ldots$ & 6 & $\ldots$ & 29 & $\ldots$ \\
\hline \multicolumn{9}{|l|}{ Smoking } \\
\hline Never & 47 & (10.3) & 502 & (40.9) & 11 & (14.3) & 335 & (29.0) \\
\hline Former & 58 & (12.7) & 215 & (17.5) & 19 & $(24.7)$ & 386 & (33.4) \\
\hline Current & 353 & (77.1) & 510 & (41.6) & 47 & $(61.0)$ & 433 & (37.5) \\
\hline Missing & 2 & $\ldots$ & 4 & $\ldots$ & 6 & $\ldots$ & 26 & $\ldots$ \\
\hline Obese & 97 & (22.6) & 382 & (31.7) & 8 & (10.5) & 100 & $(10.2)$ \\
\hline Missing & 30 & $\ldots$ & 25 & $\ldots$ & 7 & $\ldots$ & 201 & $\ldots$ \\
\hline HBsAg positive & 12 & (2.6) & 28 & (2.3) & 5 & (6.2) & 81 & (7.1) \\
\hline Missing & 2 & $\ldots$ & 12 & $\ldots$ & 2 & $\ldots$ & 42 & $\ldots$ \\
\hline \multicolumn{9}{|l|}{ Fibrosis status ${ }^{\mathrm{C}}$} \\
\hline No significant fibrosis & 211 & $(51.2)$ & 1004 & (86.0) & 14 & (29.8) & 358 & (81.0) \\
\hline Moderate fibrosis & 112 & $(27.2)$ & 103 & (8.8) & 15 & (31.9) & 59 & (13.3) \\
\hline Severe fibrosis/cirrhosis & 89 & (21.6) & 60 & (5.1) & 18 & (38.3) & 25 & (5.7) \\
\hline Missing & 48 & $\ldots$ & 64 & $\ldots$ & 36 & $\ldots$ & 738 & $\ldots$ \\
\hline \multicolumn{9}{|l|}{ ART } \\
\hline Initiated before October 2001 & 212 & $(46.1)$ & 521 & $(42.3)$ & 17 & $(20.5)$ & 513 & (43.5) \\
\hline Initiated after October 2001 & 33 & (7.2) & 269 & (21.9) & 19 & (22.9) & 268 & $(22.7)$ \\
\hline
\end{tabular}

Data are presented as no. (\%) unless otherwise indicated. One hundred two participants had missing HCV status.

Abbreviations: ART, antiretroviral therapy; HBsAg, hepatitis B virus surface antigen; HCV, hepatitis C virus; HIV, human immunodeficiency virus; IDU, injection drug use; IQR, interquartile range.

a Lower limit of detection varied over time and ranged from 500 copies $/ \mathrm{mL}$ to $20 \mathrm{copies} / \mathrm{mL}$.

${ }^{b}$ Defined as $>7$ drinks per week for women and $>14$ drinks per week for men [18].

${ }^{\circ}$ Cirrhosis defined as Fibrosis- 4 Index for Liver Fibrosis (FIB- 4 ) $\geq 3.25$ or aspartate aminotransferase-to-platelet ratio index (APRI) $\geq 1$; no significant fibrosis defined as FIB-4 $<1.45$ and APRI < 0.7 ; other combinations were classified as noncirrhotic fibrosis.

Treating only coinfected persons with severe fibrosis or cirrhosis with DAAs would lead to an expected $-0.3 \%$ (95\% CI, $-.8 \%$ to $.2 \%$ ) reduction in mortality among all $\mathrm{PWH}$, and $60 \%$ of coinfected persons would be treated. Treating the same proportion of coinfected persons at baseline without fibrosis restrictions would reduce mortality among all $\mathrm{PWH}$ by $-0.4 \%$ (95\% CI, $-1.1 \%$ to $.1 \%$; Table 3 ).

Finally, the effect of treating only coinfected persons with severe fibrosis or cirrhosis and undetectable HIV RNA was an estimated $-0.2 \%(95 \% \mathrm{CI},-.5 \%$ to $.1 \%)$ reduction in mortality risk among all $\mathrm{PWH}$, and $51 \%$ of coinfected persons would be treated. If the same proportion of coinfected persons was treated without these restrictions, the mortality risk among all PWH would decrease by $-0.4 \%$ (95\% CI, $-.9 \%$ to $.1 \%$; Table 3 ).

\section{DIscussion}

Use of common eligibility criteria for DAA treatment among people with HIV/HCV coinfection consistently yields a 


\begin{tabular}{|c|c|c|c|c|c|c|}
\hline \multirow[b]{2}{*}{ Treatment } & \multirow[b]{2}{*}{ Treated $^{a}, \%$} & \multicolumn{2}{|c|}{ Treat According to Criteria } & \multicolumn{2}{|c|}{ Treat Same \% at Random } & \multirow[b]{2}{*}{$\begin{array}{l}\text { RD: Treat According to Criteria vs Treat } \\
\text { Same, \% at Random }{ }^{d}\end{array}$} \\
\hline & & $\begin{array}{l}\text { Mortality } \\
\text { Risk, \% }\end{array}$ & $\begin{array}{c}\text { Risk } \\
\text { Difference, \% }{ }^{b}\end{array}$ & $\begin{array}{l}\text { Mortality } \\
\text { Risk, \% }\end{array}$ & $\begin{array}{c}\text { Risk } \\
\text { Difference, \% }\end{array}$ & \\
\hline No intervention & 0 & $19.2(11.0-30.4)$ & Ref & $\ldots$ & $\ldots$ & $\ldots$ \\
\hline All HCV treated & 100 & $15.4(9.0-24.2)$ & $-3.7(-9.1$ to .6$)$ & $\cdots$ & $\ldots$ & $\ldots$ \\
\hline Only HIV suppressed treated ${ }^{e}$ & $89.6(84.3-93.0)$ & $17.2(10.3-26.4)$ & $-1.9(-5.2$ to .5$)$ & $15.8(9.3-24.8)$ & $-3.3(-8.1$ to .5$)$ & $1.4(-.3$ to 3.6$)$ \\
\hline Only severe fibrosis treated ${ }^{e}$ & $59.9(44.3-69.4)$ & $17.5(10.5-27.0)$ & $-1.6(-4.7$ to .8$)$ & $16.9(9.8-26.7)$ & $-2.2(-5.6$ to .3$)$ & $0.6(.0-1.4)$ \\
\hline $\begin{array}{l}\text { Only HIV suppressed and severe } \\
\text { fibrosis treated }^{e}\end{array}$ & $51.0(37.9-59.1)$ & $18.1(10.7-28.1)$ & $-1.1(-2.8$ to .6$)$ & $17.2(10.0-27.2)$ & $-1.9(-4.7$ to .3$)$ & $0.8(-.1$ to 2.4$)$ \\
\hline
\end{tabular}

Data in parentheses indicate the $95 \%$ confidence interval.

Abbreviations: $\mathrm{HCV}$, hepatitis $\mathrm{C}$ virus; $\mathrm{HIV}$, human immunodeficiency virus; $\mathrm{RD}$, risk difference.

apercentage of all people with HIV/HCV coinfection treated with direct-acting antivirals.

${ }^{\mathrm{b}}$ Comparing treating according to criteria to no treatment.

${ }^{\mathrm{C}}$ Comparing treating the same percentage of people at random at baseline to no treatment.

${ }^{d}$ Comparing treating according to criteria to treating the same percentage of people at random at baseline.

eIncludes those who meet the criteria at baseline as well as those who progress to meet it during follow-up.

smaller decline in 10-year all-cause mortality than selecting a similar proportion of persons at random at baseline, without any eligibility criteria. Treating people at baseline would require providing treatments earlier than under the criteria we investigated and could therefore entail higher upfront costs (Supplementary Figure 3). However, the additional 10-year mortality benefit of earlier treatment is substantial. Stated differently, providing treatment to those who achieve HIV suppression and progress to severe fibrosis or cirrhosis led to treatment of $51 \%$ of coinfected persons on average. We estimated that the same 10-year mortality benefit would be achieved by treating only $28 \%$ of coinfected persons at baseline chosen randomly, nearly halving the proportion treated.

The worse mortality outcomes conferred by restrictive eligibility criteria are not surprising; these criteria were developed based on financial considerations, in contrast to evidence-based guidelines that recommend treating nearly all persons with HCV [7]. By only treating coinfected individuals who have severe fibrosis or cirrhosis, we may be intervening too late in the $\mathrm{HCV}$ disease process. At that stage of liver disease, the increased risk of adverse outcomes due to fibrosis and cirrhosis may attenuate the benefit of DAA treatment.

These findings suggest that setting treatment access policies with only short-term cost-containment in mind can cause patients unnecessary harm, and lives may have been saved if careful consideration had been used to set DAA treatment policies. These restrictive policies were likely well-intentioned, as deferring treatment may have been in anticipation of market-driven DAA price reductions that would allow wider access in the future. However, such considerations are somewhat obviated by the mortality reduction we estimated for treating patients at baseline without consideration of clinical factors. In addition, SVR has been shown to improve patient-reported outcomes [31,32], so the benefits of improved treatment allocation are expected to extend beyond improved survival.

Our population intervention effect estimates additionally incorporate the proportion of PWH who would be treated. As such, these estimates can be compared with similar estimates for other conditions in this population and can be used to properly compare interventions for different comorbidities. Similar effects should be estimated for other conditions impacting PWH to optimally allocate limited treatment resources.

Because our study mostly predates the DAA era, our estimates rely on the assumption that $\mathrm{PWH}$ who are not coinfected with HCV had the same mortality that similar people with HIV/HCV coinfection would have had with successful DAA treatment. While studies have found improved liver function and reduced risks of HCV-related complications after successful DAA treatment $[33,34]$, none have compared those who achieve SVR to those who never had HCV, and thus the validity of this assumption remains an open question. One concern with this assumption is that HCV-uninfected individuals with fibrosis must have another underlying condition affecting the liver. We controlled for several risk factors for fibrosis, such as alcohol use, injection drug use, and obesity, but others probably remain. While the effects estimated after excluding those with HBV (a known cause of fibrosis) displayed a similar pattern as those in the main analysis, other violations of our assumptions are likely.

Our study is subject to additional limitations. First, we did not model incident or recurring HCV infections (and retreatment). Multiple recent studies have estimated reinfection rates among PWH of approximately 30 per 1000 person-years [35-37]. Second, over the course of this study, HIV RNA measurement and ART regimens have improved. Of the 10888 person-visits with undetectable HIV RNA, 117 were classified based on tests with lower limits of detection $>100$ copies $/ \mathrm{mL}$. We conducted 

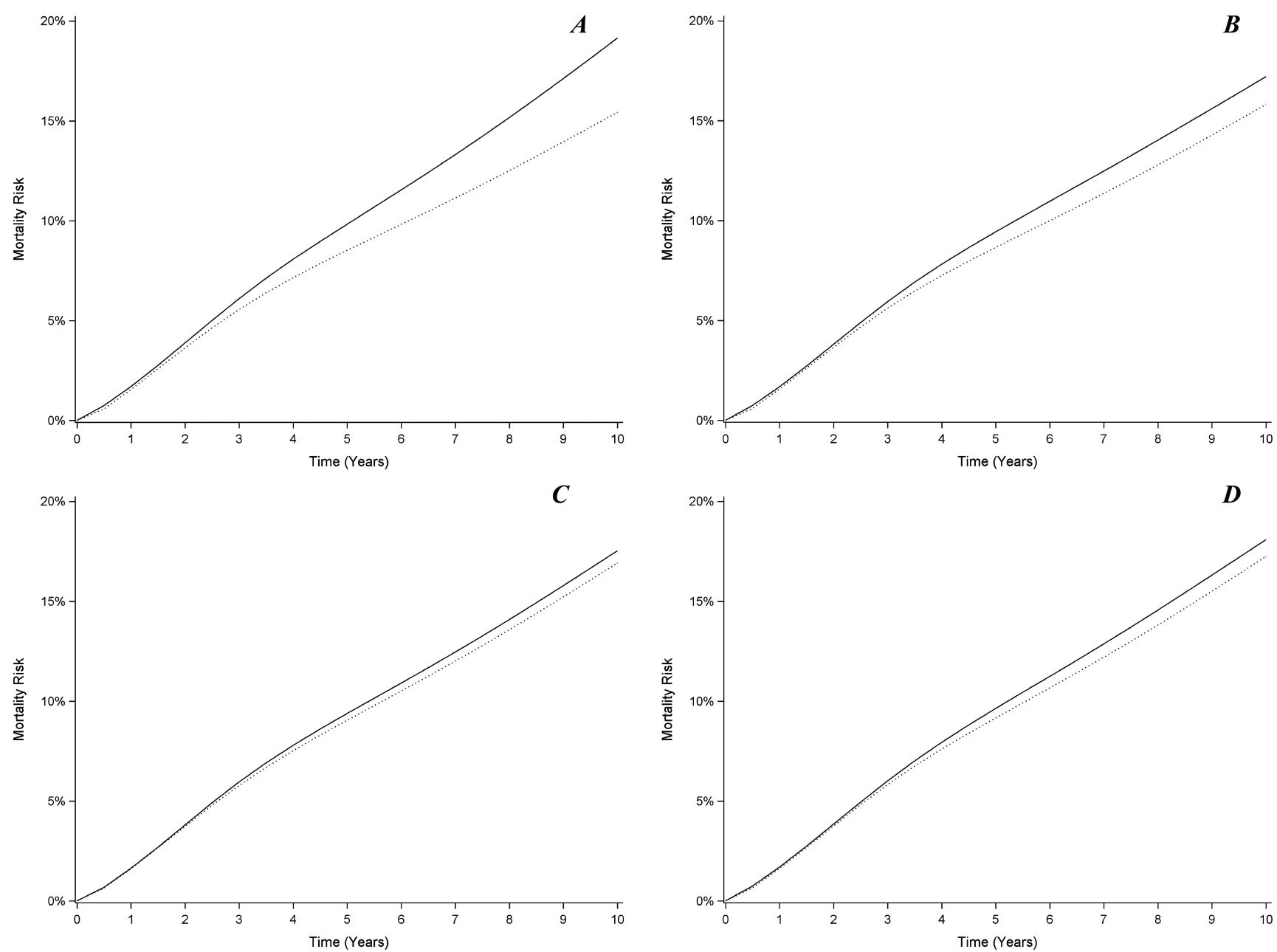

Figure 1. Ten-year risk of all-cause mortality under direct-acting antiviral treatment criteria among people with human immunodeficiency virus (HIV) and hepatitis $C$ virus in the Women's Interagency HIV Study and Multicenter AIDS Cohort Study, 1994-2015. A, Treat none at baseline (solid line) vs treat all at baseline (dotted line). B, Treat once HIV virally suppressed (solid line) vs treat same percentage of people at random at baseline (dotted line). C. Treat once progressed to severe fibrosis or cirrhosis (solid line) vs treat same percentage of people at random at baseline (dotted line). $D$, Treat once HIV virally suppressed and progressed to severe fibrosis or cirrhosis (solid line) vs treat same percentage of people at random at baseline (dotted line). Note that in all 4 panels, a lower curve indicates lower mortality, and thus in panels ( $B-D)$, treating people at random at baseline is generally superior to treating based on the stated criteria.

a sensitivity analysis classifying these measurements as detectable, and the results were unchanged. Additionally, each effect was estimated under a hypothetical intervention to have all subjects initiate "modern" ART, including those who entered the study prior to 2001, thus reducing treatment heterogeneity over the study period. Third, our study sample consisted of

Table 3. Population Intervention Effects of Direct-acting Antiviral Treatment Criteria on 10-Year All-cause Mortality Among All People With Human Immunodeficiency Virus (HIV) (Including Those With and Without Hepatitis C Coinfection), Women's Interagency HIV Study and Multicenter AIDS Cohort Study, 1994-2015

\begin{tabular}{|c|c|c|c|c|}
\hline Intervention & All HCV Treated, \% & $\begin{array}{l}\text { Only HIV Suppressed } \\
\text { Treated, \% }{ }^{\mathrm{b}}\end{array}$ & $\begin{array}{c}\text { Only Severe Fibrosis } \\
\text { Treated, \% }{ }^{\mathrm{b}}\end{array}$ & $\begin{array}{l}\text { Only HIV Suppressed and } \\
\text { Severe Fibrosis Treated, } \%^{\mathrm{b}}\end{array}$ \\
\hline$\%$ Treated $^{\mathrm{a}}$ & 100 & $89.6(84.3-93.0)$ & $59.9(44.3-69.4)$ & $51.0(37.9-59.1)$ \\
\hline RD: Treat according to criteria vs no treatment & $-0.7(-1.8$ to .1$)$ & $-0.4(-1.0$ to .1$)$ & $-0.3(-.9$ to .2$)$ & $-0.2(-.5$ to .1$)$ \\
\hline RD: Treat same $\%$ at random vs no treatment & $\ldots$ & $-0.6(-1.6$ to .1$)$ & $-0.4(-1.1$ to .1$)$ & $-0.4(-.9$ to .1$)$ \\
\hline $\begin{array}{l}\text { RD: Treat according to criteria vs treat same } \\
\% \text { at random }\end{array}$ & & $0.3(-.1$ to .7$)$ & $0.1(.0-.3)$ & $0.2(0-.4)$ \\
\hline
\end{tabular}

Data in parentheses indicate the $95 \%$ confidence interval.

Abbreviations: HCV, hepatitis C virus; HIV, human immunodeficiency virus; RD, risk difference.

aPercentage of all people with HIV/HCV coinfection treated with direct-acting antivirals.

bIncludes those who meet the criteria at baseline as well as those who progress to meet it during follow-up. 
cohorts of men who have sex with men and women in roughly even proportions, with data dating back to 1994. Our results therefore may not generalize to the current population of $\mathrm{PWH}$ in the United States [38]. Finally, because we explored the outcome of 10-year, all-cause mortality, it is expected that future changes in treatment cost and efficiency, as well as other contextual factors, will occur that may alter the effects of the policies under study.

DAA access in the United States is rapidly evolving. Though many DAA restrictions requiring patients to meet clinical preconditions are being updated [8], as of October 2018, 30\% of Medicaid programs still limit treatment to those with some degree of liver fibrosis [4], and treatment denials remain common across the country [6]. Until more person-time accumulates in the DAA era, estimates that rely on observational data, like those we present, are needed to evaluate the effects of treatment policies on long-term outcomes most pertinent to HCV, a disease that progresses slowly over decades. As we proceed further into the DAA era, studies should directly evaluate the impact of $\mathrm{HCV}$ treatment policies among people with HIV/HCV coinfection to optimize the delivery of DAAs in this population.

\section{Supplementary Data}

Supplementary materials are available at Clinical Infectious Diseases online. Consisting of data provided by the authors to benefit the reader, the posted materials are not copyedited and are the sole responsibility of the authors, so questions or comments should be addressed to the corresponding author.

\section{Notes}

Acknowledgments. Data in this manuscript were collected by the Multicenter AIDS Cohort Study (MACS) and the Women's Interagency HIV Study (WIHS). MACS (Principal Investigators): Johns Hopkins University Bloomberg School of Public Health (Joseph Margolick, Todd Brown), U01 AI35042; Northwestern University (Steven Wolinsky), U01 AI35039; University of California, Los Angeles (Roger Detels, Otoniel Martinez-Maza, Otto Yang), U01 AI35040; University of Pittsburgh (Charles Rinaldo, Lawrence Kingsley, Jeremy Martinson), U01 AI35041; the Center for Analysis and Management of MACS, Johns Hopkins University Bloomberg School of Public Health (Lisa Jacobson, Gypsyamber D'Souza), UM1 AI35043. The MACS is funded primarily by the National Institute of Allergy and Infectious Diseases (NIAID), with additional co-funding from the National Cancer Institute (NCI), the National Institute on Drug Abuse (NIDA), and the National Institute of Mental Health (NIMH). Targeted supplemental funding for specific projects was also provided by the National Heart, Lung, and Blood Institute and the National Institute on Deafness and Communication Disorders (NIDCD). MACS data collection is also supported by UL1 TR001079 (Johns Hopkins University Institute for Clinical and Translational Research [ICTR]) from the National Center for Advancing Translational Sciences (NCATS), a component of the National Institutes of Health (NIH), and NIH Roadmap for Medical Research. WIHS (Principal Investigators): UAB-MS WIHS (Mirjam-Colette Kempf and Deborah Konkle-Parker), U01 AI103401; Atlanta WIHS (Ighovwerha Ofotokun and Gina Wingood), U01 AI103408; Bronx WIHS (Kathryn Anastos and Anjali Sharma), U01 AI035004; Brooklyn WIHS (Howard Minkoff and Deborah Gustafson), U01 AI031834; Chicago WIHS (Mardge Cohen and Audrey French), U01 AI034993; Metropolitan Washington WIHS (Seble Kassaye), U01 AI034994; Miami WIHS (Margaret Fischl and Lisa Metsch), U01 AI103397; University of North Carolina (UNC) WIHS (Adaora Adimora), U01 AI103390; Connie Wofsy Women's HIV Study, Northern California (Ruth Greenblatt, Bradley Aouizerat, and
Phyllis Tien), U01 AI034989; WIHS Data Management and Analysis Center (Stephen Gange and Elizabeth Golub), U01 AI042590; Southern California WIHS (Joel Milam), U01 HD032632 (WIHS I-WIHS IV). The WIHS is funded primarily by NIAID, with additional co-funding from the Eunice Kennedy Shriver National Institute of Child Health and Human Development (NICHD), NCI, NIDA, and NIMH. Targeted supplemental funding for specific projects is also provided by the National Institute of Dental and Craniofacial Research, the National Institute on Alcohol Abuse and Alcoholism, NIDCD, and the NIH Office of Research on Women's Health. WIHS data collection is also supported by UL1 TR000004 (UCSF Clinical and Translational Science Award [CTSA]), UL1 TR000454 (Atlanta CTSA), and P30 AI050410 (UNC Center for AIDS Research). The MACS website is located at http://aidscohortstudy.org/, and the WIHS website is located at http://wihshealth.org.

Disclaimer. The contents of this publication are solely the responsibility of the authors and do not represent the official views of the NIH, Johns Hopkins ICTR, or NCATS.

Financial support. This work was supported by NIAID (grant numbers R01 AI100654 to S. R. C., P30 AI50410 to M. G. H., U01 AI35042 to C. L. T., and K24 AI108516 to P. C. T.) and NICHD (grant number DP2 HD084070 to D. W. and A. B.).

Potential conflicts of interest. A. A. A. has received research funding from Gilead and personal fees from ViiV, and is on a Merck advisory board. C. B. H. supervised a clinical trial under an institutional contract between UNC and AbbVie. P. C. T. is conducting investigator-initiated research sponsored by Merck. C. L. T. has received research funding paid to her institution from Gilead Sciences and Sanofi. All other authors report no potential conflicts. All authors have submitted the ICMJE Form for Disclosure of Potential Conflicts of Interest. Conflicts that the editors consider relevant to the content of the manuscript have been disclosed.

\section{References}

1. Collins S, Mertenskoetter T, Loeliger E, et al. Liver-related deaths in persons infected with the human immunodeficiency virus: the D:A:D Study. Arch Intern Med 2006; 166:1632-41.

2. Weber R, Ruppik M, Rickenbach M, et al; Swiss HIV Cohort Study. Decreasing mortality and changing patterns of causes of death in the Swiss HIV Cohort Study. HIV Med 2013; 14:195-207.

3. Milazzo L, Lai A, Calvi E, et al. Direct-acting antivirals in hepatitis $C$ virus (HCV)-infected and HCV/HIV-coinfected patients: real-life safety and efficacy. HIV Med 2017; 18:284-91.

4. National Viral Hepatitis Roundtable, Harvard Law School Center for Health Law and Policy Innovation. Hepatitis C: the state of Medicaid access. 2017. Available at: https://stateofhepc.org/wp-content/uploads/2017/10/State-of-HepC_2017_ FINAL.pdf. Accessed 2 April 2018.

5. Ooka K, Connolly JJ, Lim JK. Medicaid reimbursement for oral direct antiviral agents for the treatment of chronic hepatitis C. Am J Gastroenterol 2017; 112:828-32.

6. Gowda C, Lott S, Grigorian M, et al. Absolute insurer denial of direct-acting antiviral therapy for hepatitis $\mathrm{C}$ : a national specialty pharmacy cohort study. Open Forum Infect Dis 2018; 5: ofy076.

7. American Association for the Study of Liver Diseases/Infectious Diseases Society of America. HCV guidance: recommendations for testing, managing, and treating hepatitis C. 2017. Available at: https://www.hcvguidelines.org/evaluate/whenwhom. Accessed 13 February 2018.

8. Kapadia SN, Jeng PJ, Schackman BR, Bao Y. State Medicaid hepatitis C treatment eligibility criteria and use of direct-acting antivirals. Clin Infect Dis 2018; 66:1618-20.

9. Kirk GD, Mehta SH, Astemborski J, et al. HIV, age, and the severity of hepatitis C virus-related liver disease: a cohort study. Ann Intern Med 2013; 158:658-66.

10. Kaslow RA, Ostrow DG, Detels R, Phair JP, Polk BF, Rinaldo CR Jr. The Multicenter AIDS Cohort Study: rationale, organization, and selected characteristics of the participants. Am J Epidemiol 1987; 126:310-8.

11. Barkan SE, Melnick SL, Preston-Martin S, et al. The Women's Interagency HIV Study. WIHS collaborative study group. Epidemiology 1998; 9:117-25.

12. Adimora AA, Ramirez C, Benning L, et al. Cohort profile: the Women's Interagency HIV Study (WIHS). Int J Epidemiol 2018; 47:393-94i.

13. Sterling RK, Lissen E, Clumeck $\mathrm{N}$, et al; APRICOT Clinical Investigators. Development of a simple noninvasive index to predict significant fibrosis in patients with HIV/HCV coinfection. Hepatology 2006; 43:1317-25. 
14. Lin ZH, Xin YN, Dong QJ, et al. Performance of the aspartate aminotransferase-to-platelet ratio index for the staging of hepatitis C-related fibrosis: an updated meta-analysis. Hepatology 2011; 53:726-36.

15. Panel on Antiretroviral Guidelines for Adults and Adolescents. Guidelines for the use of antiretroviral agents in adults and adolescents living with HIV. Department of Health and Human Services. 2014. Available at: https://aidsinfo.nih.gov/contentfiles/AdultandAdolescentGL003392.pdf. Accessed 18 January 2018.

16. Panel on Antiretroviral Guidelines for Adults and Adolescents. Guidelines for the use of antiretroviral agents in HIV-1-infected adults and adolescents. Department of Health and Human Services. 2018. Available at: http://aidsinfo.nih.gov/contentfiles/lvguidelines/AdultandAdolescentGL.pdf. Accessed 4 April 2018.

17. Greenland S, Pearl J, Robins JM. Causal diagrams for epidemiologic research. Epidemiology 1999; 10:37-48.

18. US Department of Health and Human Services. Appendix 9: alcohol. 2015 Available at: https://health.gov/dietaryguidelines/2015/guidelines/appendix-9/. Accessed 19 March 2018.

19. Hubbard AE, Laan MJ. Population intervention models in causal inference. Biometrika 2008; 95:35-47.

20. Westreich D. From exposures to population interventions: pregnancy and response to HIV therapy. Am J Epidemiol 2014; 179:797-806.

21. Westreich D. From patients to policy: population intervention effects in epidemiology. Epidemiology 2017; 28:525-8.

22. Robins J. A new approach to causal inference in mortality studies with a sustained exposure period-application to control of the healthy worker survivor effect. Math Model 1986; 7:1393-12.

23. Robins JM, Hernán MA. Estimation of the causal effects of time-varying exposures. In: Longitudinal data analysis. New York: Chapman and Hall/CRC Press, 2009: 553-99.

24. Westreich D, Cole SR, Young JG, et al. The parametric g-formula to estimate the effect of highly active antiretroviral therapy on incident AIDS or death. Stat Med 2012; 31:2000-9.

25. Keil AP, Edwards JK, Richardson DB, Naimi AI, Cole SR. The parametric g-formula for time-to-event data: intuition and a worked example. Epidemiology 2014; 25:889-97.

26. Naggie S, Cooper C, Saag M, et al; ION-4 Investigators. Ledipasvir and sofosbuvir for HCV in patients coinfected with HIV-1. N Engl J Med 2015; 373:705-13.
27. Efron B, Tibshirani R. Bootstrap methods for standard errors, confidence intervals, and other measures of statistical accuracy. Stat Sci 1986; 1:54-75.

28. Allison PD. Missing data. Thousand Oaks, CA: Sage Publications, 2001.

29. Schomaker M, Heumann C. Bootstrap inference when using multiple imputation. Stat Med 2018; 37:2252-66.

30. Westreich D, Mooney S. Number (of whom) needed to treat (with what). Epidemiology. In press.

31. Younossi ZM, Stepanova M, Sulkowski M, Naggie S, Henry L, Hunt S. Sofosbuvir and ledipasvir improve patient-reported outcomes in patients co-infected with hepatitis $\mathrm{C}$ and human immunodeficiency virus. J Viral Hepat 2016; 23:857-65.

32. Marcellin F, Roux P, Protopopescu C, Duracinsky M, Spire B, Carrieri MP. Patientreported outcomes with direct-acting antivirals for the treatment of chronic hepatitis C: current knowledge and outstanding issues. Expert Rev Gastroenterol Hepatol 2017; 11:259-68.

33. Cheung MCM, Walker AJ, Hudson BE, et al. Outcomes after successful direct-acting antiviral therapy for patients with chronic hepatitis $\mathrm{C}$ and decompensated cirrhosis. J Hepatol 2016; 65:741-47. Available at: https:// www.sciencedirect.com/science/article/pii/S0168827816303142. Accessed 28 November 2018.

34. Foster GR, Irving WL, Cheung MCM, et al. Impact of direct acting antiviral therapy in patients with chronic hepatitis $\mathrm{C}$ and decompensated cirrhosis. J Hepatol 2016; 64:1224-31. Available at: https://www.sciencedirect.com/science/ article/pii/S0168827816000659. Accessed 28 November 2018.

35. Islam N, Krajden M, Shoveller J, et al; British Columbia Hepatitis Testers Cohort Team. Incidence, risk factors, and prevention of hepatitis $\mathrm{C}$ reinfection: a population-based cohort study. Lancet Gastroenterol Hepatol 2017; 2:200-10.

36. Young J, Rossi C, Gill J, et al; Canadian Co-infection Cohort Investigators. Risk factors for hepatitis $\mathrm{C}$ virus reinfection after sustained virologic response in patients coinfected with HIV. Clin Infect Dis 2017; 64:1154-62.

37. Rossi C, Butt ZA, Wong S, et al; BC Hepatitis Testers Cohort Team. Hepatitis C virus reinfection after successful treatment with direct-acting antiviral therapy in a large population-based cohort. J Hepatol 2018; 69:1007-14.

38. Lesko CR, Cole SR, Hall HI, et al. The effect of antiretroviral therapy on all-cause mortality, generalized to persons diagnosed with HIV in the USA, 2009-11. Int J Epidemiol 2016; 45:140-50. 